\title{
Thermal Convection and Emergence of Isolated Vortices in Soap Bubbles
}

\author{
F. Seychelles, ${ }^{1}$ Y. Amarouchene, ${ }^{1}$ M. Bessafi, ${ }^{2}$ and H. Kellay ${ }^{1}$ \\ ${ }^{1}$ Université Bordeauxl, CPMOH UMR 5798 du CNRS, 351 cours de la Libération, 33405 Talence France \\ ${ }^{2}$ Université de la Réunion, Laboratoire de Génie Industriel, 15 avenue René Cassin, 97715 Sanit Denis, Réunion, France
}

(Received 27 July 2007; published 7 April 2008)

A novel thermal convection cell consisting of half a soap bubble heated at the equator is introduced to study thermal convection and the movement of isolated vortices. The soap bubble, subject to stratification, develops thermal convection at its equator. A particular feature of this cell is the emergence of isolated vortices. These vortices resemble hurricanes or cyclones and similarities between our observed structures and these natural objects are found. This is brought forth through a study of the mean square displacement of these objects showing signs of superdiffusion.

DOI: 10.1103/PhysRevLett.100.144501

Turbulent thermal convection which is ubiquitous in several natural settings such as the atmosphere or the inner core of planets, has attracted and continues to attract considerable attention from experimentalists and theorists. Experiments have demonstrated several robust features of this phenomenon, in closed containers heated from below, such as the importance of thermal plumes and the onset of a large scale circulation. Most experiments use three dimensional geometries but a few recent experiments have demonstrated similar features in two dimensions $[1,2]$ by using vertical, stably stratified, soap films as two dimensional fluids since the flow occurs in the plane of the film. Soap films have now become model systems to study two dimensional hydrodynamics and turbulence [3] and these recent experiments extend their use to turbulent thermal convection. Interest in two dimensional turbulence stems from the fact that atmospheric turbulence at large scales displays two dimensional features due to the small thickness of the Earth's atmosphere [4]. A specific feature is the emergence and persistence of isolated vortices. The great red spot of Jupiter has been brought forth as a sign of the two dimensional nature of atmospheric turbulence [5]. We here take advantage of both the two dimensional nature of soap films and the fact that they can be rendered turbulent through thermal convection to study turbulent soap bubbles and therefore mimic a curved two dimensional space such as the atmosphere. The soap bubble (actually a half bubble is used) is heated at the equator giving rise to thermal convection. A prominent feature of our new setup is the emergence of long lived isolated vortices reminiscent of natural ones such as the red spot or hurricanes and cyclones. A major difference between our two dimensional setup and previously used cells is the absence of lateral walls. We believe that this absence of walls is at the origin of the emergence of long lived isolated vortices as opposed to a large scale circulation in cells with lateral walls.

Figure 1 shows typical half bubbles heated at the equator at different temperatures and illuminated with white light. Interference colors mark the surface of the bubble indicating variations in the thickness of the soap film. When no
PACS numbers: 47.55.pb, 47.27.-i, 47.32.C-, 47.55.Hd

temperature gradient or a small gradient is present the thickness of the bubble decreases as the height increases giving rise to the horizontal bands seen in the photograph. The two dimensional density of the soap film being $\rho h$, where $\rho$ is the density of soap water and $h$ its thickness, the film is stably stratified with dense fluid at the bottom and lighter fluid at the top. When a sufficient temperature gradient is applied, the region near the equator is host to rising plumes just like in conventional thermal convection. This convection zone extends all around the equator and grows in height as the gradient increases as shown in Fig. 1. The upper part of the bubble is more quiescent than the zone near the equator. We first study the properties of the convective zone near the equator by measuring the statistics of the velocity and thickness fluctuations and compare them to previous measurements in flat cells with lateral walls [2]. No particular difference appears at this stage. A major difference is the absence of a large scale circulation and the emergence and persistence of single isolated vortices in the upper part of the half bubble as displayed in

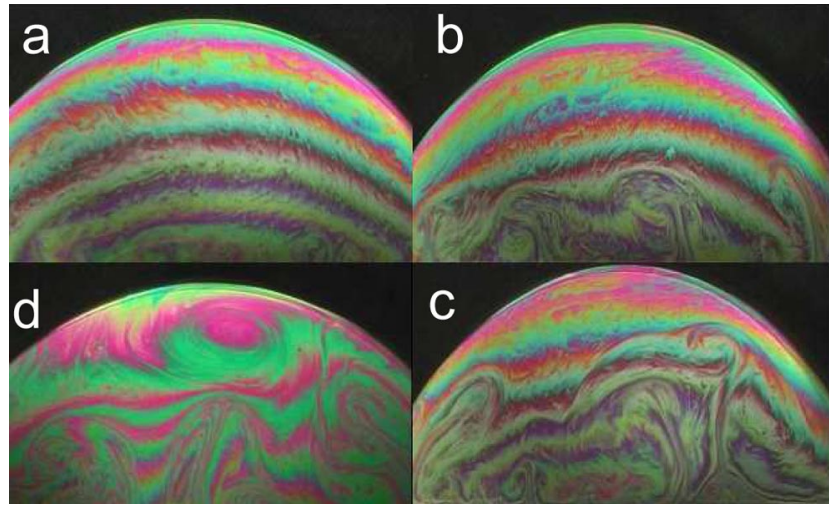

FIG. 1 (color online). Images of bubbles at different temperature gradients. $\Delta T$ increases from a to $c$ with $\Delta T=9,17$ and $31{ }^{\circ} \mathrm{C}$ respectively. The convection zone grows in extent as $\Delta T$ increases. Plumes can be seen in this zone. Image $\mathrm{d}$ : a bubble with a convection zone and an isolated vortex near the top for $\Delta T=45^{\circ} \mathrm{C}$. 
Fig. 1(d). Their presence is more frequent as the temperature difference increases. The mean square displacement of these vortices varies as a power law in time. This scaling is different from the one expected for diffusive behavior and shows signs of superdiffusion. Surprisingly, analysis of the trajectories of natural hurricanes in the Earth's atmosphere gives rise to a similar scaling law for their mean square displacement versus time. Thus, the properties of our isolated vortices in this novel experimental system mimic some features of the position fluctuations of natural hurricanes. This suggests that a small experimental setup such as ours may allow a careful study of such large scale phenomena of importance for atmospheric science and for meteorology.

Our setup consists of a hollow brass ring with an inlet and outlet for water circulation to thermostat the full apparatus at the desired temperature. This brass ring has a circular slot which can be filled with soap water. The middle part of the ring was covered with a Teflon disk. The half bubble was blown with a straw using the soap solution in the circular slot. The ring could be kept at the desired temperature to a precision of $\pm 0.1^{\circ} \mathrm{C}$ using the water circulation thermostat. The temperature of the solution in contact with the ring and the temperature at the top of the bubble were measured using a needlelike thermistor. The temperature difference between the bottom and the top parts of the half bubble will be denoted $\Delta T$ which is our control parameter. The room was kept at a constant temperature of $17^{\circ} \mathrm{C}$. The difference in temperature $\Delta T$ can be changed in the range 5 to $45^{\circ} \mathrm{C}$. The ring has two concentric slots so we could vary the diameter of the half bubble which could be fixed to either 8 or $10 \mathrm{~cm}$. Typical half bubbles with strong convective patterns are shown in Fig. 1. The patterns are filmed using a 3CCD camera. The velocity in a single point is measured using an LDV capable of measuring both the horizontal and vertical components of the velocity vector. The soap solution is water at different concentrations $c$ of detergent ranging from 0.2 to $5 \%$. This solution was seeded with $0.3 \mu \mathrm{m}$ diameter polystyrene beads for the velocity measurements. The interference images of the soap film are digitized and analyzed using image analysis software. The interference colors are converted to thickness using a suitable calibration. We analyzed the thickness $h$ field by extracting the second order structure functions $\left\langle\delta h^{2}(r)\right\rangle=\langle(h(r)-$ $\left.h(0))^{2}\right\rangle$, where the brackets indicate a spatial average over the whole field of view and an average over several images and $r$ is the spatial increment (which could be either in the horizontal direction $\delta r_{x}$ or in the vertical direction $\delta r_{y}$ ). Images of the isolated vortices were recorded with a fast camera. The position of the center of the vortex could be tracked with a temporal resolution of $4 \mathrm{~ms}$.

The properties of the convective zone share some similarities with those observed in experiments of convection in vertical soap films. A common feature is the scaling of the velocity field. The results of Zhang and $\mathrm{Wu}$ [2] show that in the turbulent regime obtained for high $\Delta T$, the second order structure function of the velocity differences scales as $\left\langle\delta v^{2}(r)\right\rangle=\left\langle(v(r)-v(0))^{2}\right\rangle \sim r$. This scaling is consistent with Bogliano's prediction [6] for the energy density spectrum which reads: $E(k) \sim k^{-11 / 5}$. Our results for the velocity spectra are displayed in Fig. 2. The horizontal axis is frequency because the one point measurements are time series of the velocity. The frequency axis can be converted to a wave number in the direction of the mean flow using Taylor's frozen turbulence assumption. The mean velocity here is horizontal so the wave number is in the horizontal direction and is given by $k_{x}=$ $2 \pi f / V_{\text {mean }}$. A histogram of the horizontal velocity fluctuations is shown in the inset to Fig. 2 showing a well-defined nonzero velocity. The scaling obtained from our data gives an exponent of -2.2 which is consistent with the findings of Zhang and $\mathrm{Wu}$ [2] and is in agreement with the Bogliano scaling expected for the buoyancy subrange of turbulence in stably stratified fluids [6]. The other result we have obtained concerns the structure functions of thickness differences across a scale $r$ shown in Fig. 3. For low $\Delta T$, the structure function shows a scaling law with an exponent close to 0.4 . This scaling is consistent with previous results [2] for small temperature differences and is consistent with the Bogliano regime for which an exponent of 0.4 is expected [6]. For high enough $\Delta T$, the structure functions change and become almost flat versus the scale $r$ as expected for the so-called Batchelor regime [7,8]. Zhang and $\mathrm{Wu}[2]$ also observed a similar transition for the behavior of the thickness field as $\Delta T$ increases which they have attributed to the onset of a large scale circulation in the cell accompanied by an inversion of the stratification from stable to unstable. No density inversion is observed here but a marked reduction (by a factor 4) of the density

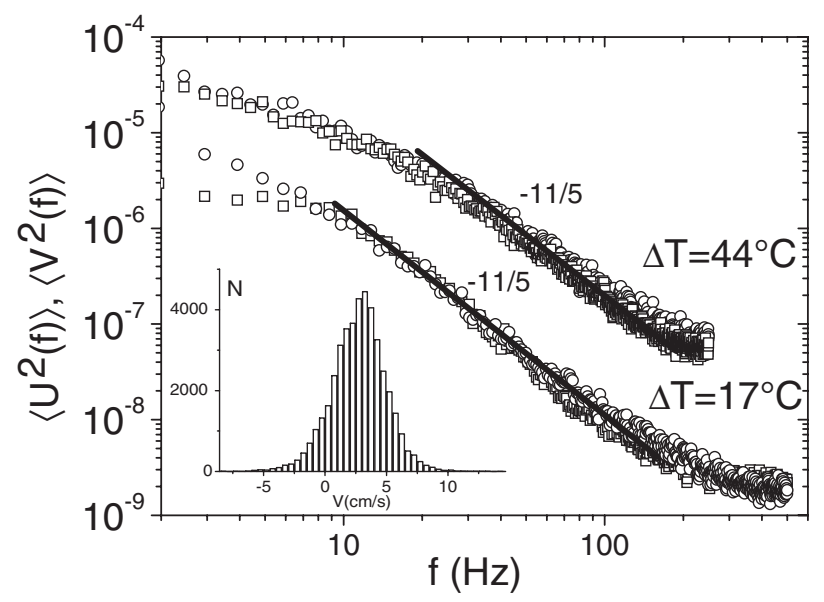

FIG. 2. Velocity spectra in the convection zone. Circles are for the vertical velocity while squares are for the horizontal velocity. The solid line is the Bogliano prediction. Inset: a histogram of the horizontal velocity fluctuations for $\Delta T=44{ }^{\circ} \mathrm{C}$. 


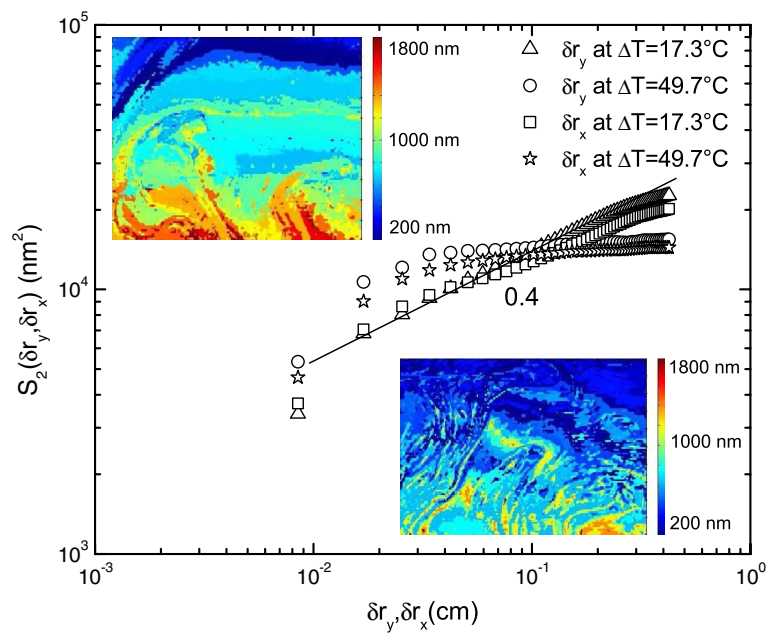

FIG. 3 (color online). Second order structure functions of the thickness fluctuations in the convection zone. Insets: thickness fields for the lower $\Delta T$ (upper) and the higher $\Delta T$ (bottom).

gradient is observed as $\Delta T$ increases. Also, strong mixing is noticeable at high $\Delta T$ as seen in Fig. 1. Convection in this novel setup without lateral walls turns out to display similar statistical features as in flat films studied earlier. So far, the role of the curvature of the film does not appear. The major difference with flat films, where a large scale circulation has been reported [2], seems to be the emergence of isolated and persistent vortices in the upper half of the soap bubble.

Such a vortex is shown in Fig. 1(d). These isolated vortices emerge randomly on the surface of the bubble, grow in size rapidly as illustrated in Fig. 4, and persist for relatively long times almost equivalent to the lifetime of the half bubble itself which could last several minutes. The occurrence of these vortices becomes more probable for higher $\Delta T$ while they are almost absent for small $\Delta T$. At first sight, these isolated vortices move around the bubble randomly. A typical trajectory is shown in Fig. 5. These vortices move around the bubble with velocities near $1 \mathrm{~cm} / \mathrm{s}$. We analyze these trajectories by calculating the

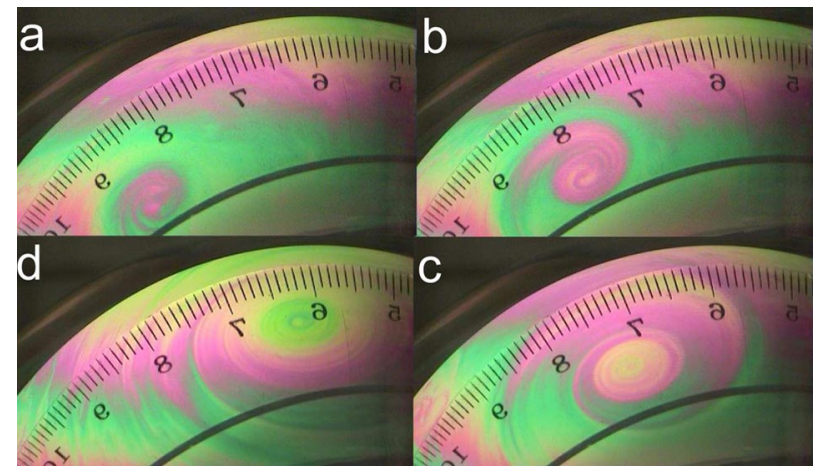

FIG. 4 (color online). Birth and growth of a single vortex. The time between successive images is $0.16 \mathrm{~s}, 0.44 \mathrm{~s}$, and $0.76 \mathrm{~s}$. The image of a transparent ruler (in $\mathrm{cm}$ ) was projected on the bubble. mean square displacement $\left\langle r^{2}(\tau)\right\rangle=\left\langle(r(t+\tau)-r(t))^{2}\right\rangle$ for different time increments $\tau$. This analysis shows that $\left\langle r^{2}(\tau)\right\rangle \sim \tau^{\alpha}$ with $\alpha \sim 1.6$. This scaling law is to be contrasted with Brownian motion for which the scaling exponent is 1 . Figure 5 summarizes this result for different temperature differences $\Delta T$. Here we plot $\frac{\left\langle r^{2}(\tau)\right\rangle}{\left\langle r^{2}\left(\tau_{c}\right)\right\rangle}$ versus $\frac{\tau}{\tau c}$. The characteristic time $\tau_{c}$ is the correlation time obtained from the correlation function $\langle r(t+\tau) r(t)\rangle$. The rescaling by $\tau_{c}$ and the corresponding mean squared displacement $\left\langle r^{2}\left(\tau_{c}\right)\right\rangle$ collapses all of our data for different temperature differences $\Delta T$. The scaling law we observe is valid for more than a decade in time scales below $\tau_{c}$. Above $\tau_{c}$ the mean square displacement seems to flatten with no systematic dependence. To our knowledge this is the first time that an isolated vortex is tracked in a turbulent 2D medium. Despite the complexity of the problem, a single scaling law summarizes all of our data and strongly suggests that these vortices are superdiffusive, a behavior observed here for the first time as far as we know. Super diffusion arises in so called Levy flights [9] for which the spatial steps for a fixed time increment are distributed according to a power law $P d f(\delta r) \sim r^{-\beta}$. The exponent of this power law is directly related to the exponent $\alpha$ by the relation $\beta=1+2 / \alpha$. A $P d f$ of the displacements $\Delta x$ and $\Delta y$ in the horizontal and vertical directions is shown in the inset to Fig. 5. A small power law range can be observed here with an exponent $\beta=2.2$ in good agreement with that obtained from the mean square displacement as indicated by the solid line. This indicates that the movement of the vortices can be recast into the random walks known as Levy flights.

Isolated vortices occur in natural settings as well. Because of the similarities between our isolated vortices and hurricanes or cyclones we have analyzed the trajectories of certain natural hurricanes along similar lines. It

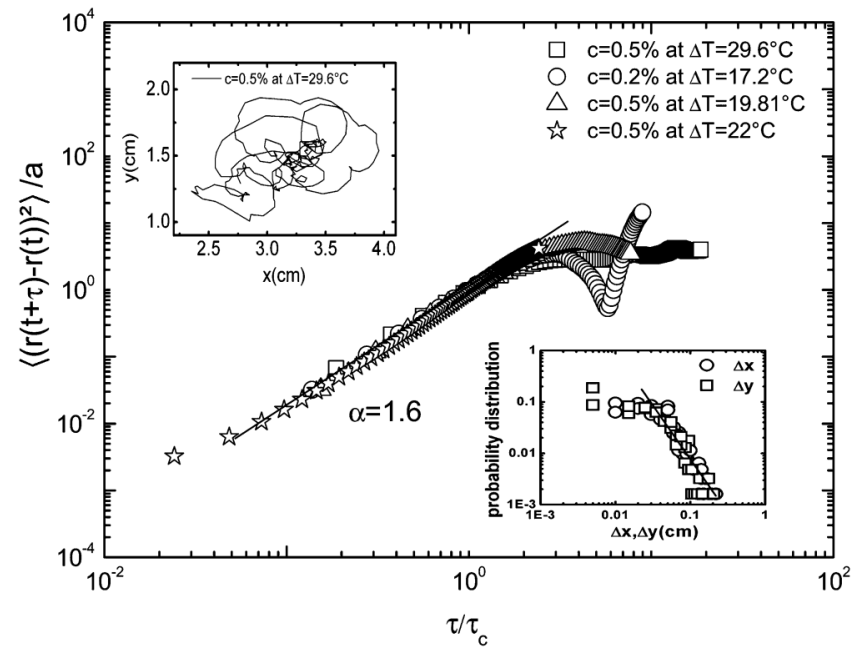

FIG. 5. Mean square displacement of the isolated vortices for different $\Delta T$. Upper inset: A track of an isolated vortex. Lower inset: the $P d f$ of the increment $\Delta x$ and $\Delta y$ for a fixed time interval $\Delta t$. 


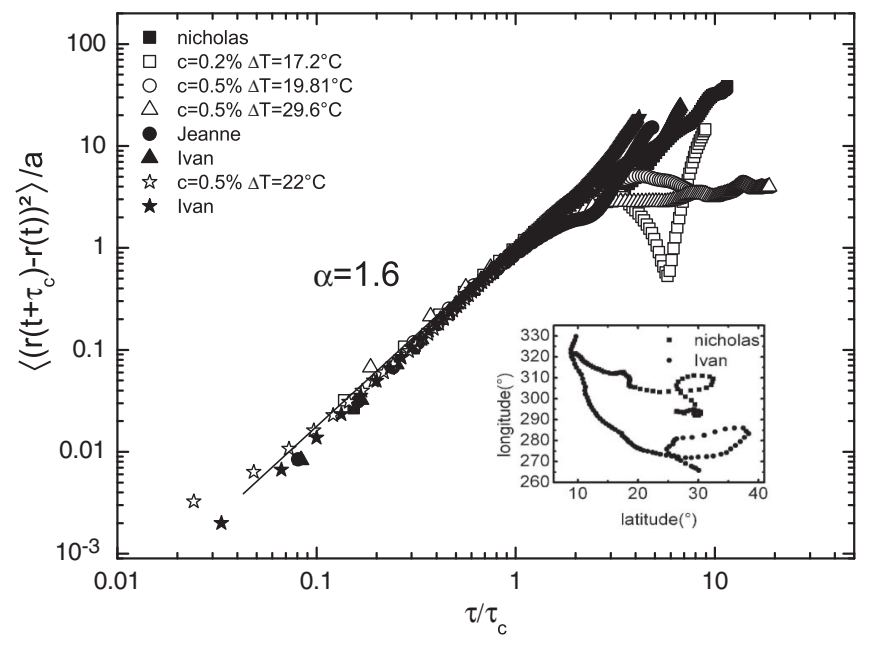

FIG. 6. Mean squared displacement for natural hurricanes plotted along with some of the data from the isolated vortices in this experiment. Lower inset: Two hurricane tracks.

should be signalled here that natural hurricanes seem to travel along relatively well defined mean trajectories for which the Coriolis force and the beta-effect play a central role. However, they do show fluctuations around this mean trajectory. An analysis of the mean square displacement of different hurricanes including Nicholas (2003), Jeanne (2004), and Ivan (2004) show a very similar behavior as our isolated vortices. The hurricane trajectories were obtained from the National Hurricane center web site and consist of either satellite observations or of radar data. Their trajectories are sampled every 6 hours for satellite data and every 15 minutes for radar data. The analysis of these trajectories is summarized in Fig. 6 where the trajectories are displayed in the inset. Here, the data of Fig. 5 are replotted so as to illustrate the similarity. This plot shows that the hurricanes and our isolated vortices display very similar features especially the power law scaling at times smaller than $\tau_{c}$. The scaling exponent turns out to be very close to the value extracted from our vortices namely $\alpha=1.6$.

Super diffusive behavior can be traced to a non trivial interaction between the moving object and the medium. An example of entities that interact with the medium itself has been illustrated through a study of the superdiffusion of passive beads in a bath of self propelling bacteria [10]. Another example concerns the movement of passive beads in a laminar rotating flow where vortices may trap and release the particles giving rise to superdiffusion [11]. The isolated vortices here are kicked by the turbulent agitation of the surrounding flow. These vortices, being part of the flow, must have an important reaction on the medium itself. In addition, the movement of vortices is sensitive to the sign of vorticity gradients [12] which in a turbulent medium may show a complicated spatial and temporal distribution and a non trivial interaction with the moving vortex giving rise to a complex trajectory and dynamics.

In conclusion, we have introduced a novel setup to study thermal convection in a quasi two dimensional curved geometry. While the statistics of the velocity and scalar fluctuations appear to be similar to those in flat films, our experiments show that the curved nature of the bubble used allows for isolated vortices to emerge. The absence of walls is the most probable reason for the emergence of such structures. These isolated vortices resemble natural hurricanes for certain aspects. In particular, when the mean squared displacement of the eye is examined superdiffusion is recovered. This superdiffusion is probably indicative of Levy flights and calls for further theoretical work on the movement of isolated vortices in a turbulent medium which is important for turbulence in general and for atmospheric and meteorological studies, in particular.

We thank H. Tarbague for help with the initial stages of this experiment.

[1] B. Martin and X. L. Wu, Phys. Rev. Lett. 80, 1892 (1998).

[2] J. Zhang, X. L. Wu, and K. Q. Xia, Phys. Rev. Lett. 94, 174503 (2005); J. Zhang and X. L. Wu, Phys. Rev. Lett. 94, 234501 (2005).

[3] H. Kellay and W. I. Goldburg, Rep. Prog. Phys. 65, 845 (2002).

[4] P. Morel and M. Larcheveque, J. Atmos. Sci. 31, 2189 (1974).

[5] P. S. Marcus, Nature (London) 428, 828 (2004).

[6] R. Bolgiano, J. Geophys. Res. 64, 2226 (1959).

[7] G. K. Batchelor, J. Fluid Mech. 5, 113 (1959).

[8] Y. Amarouchene and H. Kellay, Phys. Rev. Lett. 93, 214504 (2004).

[9] Levy Flights and Related Topics in Physics, edited by M.F. Shlesinger, G. M. Zaslavsky, and U. Frisch, Lecture Notes in Physics (Springer-Verlag, Berlin, 1995); J. P. Bouchaud and A. George, Phys. Rep. 195, 127 (1990).

[10] X.L. Wu and A. Libchaber, Phys. Rev. Lett. 84, 3017 (2000).

[11] T.H. Solomon, E. R. Weeks, and H. L. Swinney, Phys. Rev. Lett. 71, 3975 (1993).

[12] D. A. Schecter and D. H.E. Dubin, Phys. Rev. Lett. 83, 2191 (1999). 